\title{
Renuencia a hablar en clase y estilos de aprendizaje entre los estudiantes de ELE en Taiwán
}

\author{
JORGE DANIEL MENDOZA PUERTAS \\ Universidad Providence \\ jmenpue8@pu.edu.tw
}

\section{Resumen}

En el campo de la enseñanza-aprendizaje de lenguas extranjeras, la renuencia a hablar en clase es un fenómeno complejo y poliédrico que dificulta el adecuado desarrollo de la competencia comunicativa. Este fenómeno, con gran presencia en las aulas de Asia Oriental, ha quedado tradicionalmente vinculado a la imagen de un estudiante poco participativo, silencioso y con preferencia por estilos de aprendizaje pasivos. En este trabajo, a partir de la aplicación de dos cuestionarios, la Reticence Scale-12 y el CHAEA, analizamos el fenómeno de la renuencia y las preferencias de aprendizaje de los estudiantes taiwaneses de ELE sobre una muestra de alumnos universitarios. Posteriormente, empleando correlaciones estadísticas, observamos si existe alguna relación entre este rechazo a hablar en clase y los distintos estilos de aprendizaje. Los resultados obtenidos revelan un importante número de aprendientes renuentes a comunicarse, así como una preferencia mayoritariamente moderada por todos los estilos a excepción del estilo reflexivo. Por este último, en contra de lo que sería esperable, la mayoría de los encuestados presenta una preferencia baja. Por último, las pruebas estadísticas revelan correlaciones negativas y significativas pero bajas entre los estilos activo y pragmático y la renuencia a hablar en clase.

\section{Palabras clave}

Renuencia a hablar; estilos de aprendizaje; ELE; universidad; Taiwán. 


\title{
Reluctance to speak and learning styles among SFL students in Taiwan
}

\begin{abstract}
In the field of foreign language learning and teaching, the reluctance to speak in class is a complex and polyhedral phenomenon hinders the proper development of communication skills. This phenomenon, with a great presence in East Asian classrooms, has traditionally been linked to the image of a student who are not very participating, silent and with a preference for passive learning styles. In this work, based on the application of two questionnaires, the Reticence Scale-12 and the CHAEA, we analyse the phenomenon of communicative reticence and learning preferences of Taiwanese SFL learners over a sample of university students. Later, using statistical correlations, we observe if there is any relationship between the refusal to speak in class and the different learning styles. The results obtained reveal a significant number of learners are reluctant to communicate. Along with a mostly moderate preference for all styles except the reflective style. For the latter, the majority of the respondents present a low preference, contrary to what would be expected. Finally, statistical tests reveal negative and significant, but low correlations between active and pragmatic styles and reluctance to speak in class.
\end{abstract}

\section{Keywords}

Reticence; learning styles; SFL; undergraduate students; Taiwan.

Recibido el 23/11/2020

Aceptado el 18/06/2021 


\section{Introducción y marco teórico}

Actualmente nadie duda de que la práctica oral en la clase de lenguas extranjeras influye positivamente en el aprendizaje de la lengua así como en el desarrollo de la competencia comunicativa (Arnold y Fonseca, 2004). No en vano, como ya expuso Swain (1995), el output o producción oral posee una serie de funciones propias e independientes a las de la comprensión auditiva o input, y estas son indispensables para avanzar en el dominio de cualquier lengua. El paso del procesamiento semántico al sintáctico, la comprobación de hipótesis, la reflexión sobre la propia lengua y la mejora de la fluidez constituyen sus principales beneficios. Sin embargo, la renuencia a hablar en clase, fenómeno ampliamente documentado en las aulas de todo el mundo (Talley y $\mathrm{Tu}, 2015)$, dificulta el progreso en el aprendizaje minimizando las posibilidades de desarrollar unas adecuadas habilidades comunicativas. Este rechazo a hablar no es nada nuevo para los profesionales que ejercen la docencia en países de Asia Oriental, donde este fenómeno ha recibido una especial atención en el ámbito de la enseñanza del inglés: el desajuste existente entre la práctica del enfoque comunicativo, imperante en las últimas décadas, y el perfil poco participativo del alumnado ha hecho surgir numerosas investigaciones al respecto. No obstante, en el campo de la enseñanza del español son pocos los trabajos en los que podemos encontrar un tratamiento integral de este fenómeno ${ }^{1}$.

El carácter multidimensional o poliédrico con el que ha sido definida la renuencia a hablar revela su extraordinaria complejidad. Las razones identificadas por investigadores y profesores han sido tan numerosas como variadas, por lo que se ha alcanzado un amplio consenso acerca de los muchos factores que influyen sobre el individuo que ofrece esta resistencia. Entre ellos encontramos elementos que van desde aquellos de índole afectiva o psicológica hasta aquellos de simple índole contextual, pasando por otros de carácter pedagógico, lingüístico y sociocultural (Bao, 2014; Chang, 2011; Hsieh, 2015, 2016; Jackson, 2003; Jenkins, 2008; Liu, 2005; Mendoza, 2016, 2017, 2021; Shimizu, 2006; Wu, 2019)2. Algunos investigadores como Cortazzi y Jin (1996), Jones (1999), Flowerdew, Miller y Li (2000), Xiao (2007) o Bao (2014) ya apuntaron hacia los estilos de aprendizaje dominantes en estas naciones como causa que favorecería la baja participación y el rechazo a hablar en clase. Estas preferencias de aprendizaje, según señalan los autores previos, se encontrarían cultural y pedagógicamente influenciadas. En esta línea, hoy en día es sabido que los estilos de aprendizaje, además de presentar un componente hereditario o natural (Kolb, 1984), se encuentran influenciados tanto por la cultura (Auyeung y Sands, 1996; Joy y Kolb, 2008;

\footnotetext{
${ }^{1}$ La mayoría de los trabajos existentes abordan aspectos aislados que inciden sobre la resistencia a hablar en clase, pero no una visión global del fenómeno. Por ejemplo, en el ámbito sinohablante, encontramos trabajos como los de Hsieh $(2015,2016)$ con estudiantes taiwaneses y los de Muñoz Torres $(2010 \mathrm{a}, 2010 \mathrm{~b})$ con estudiantes de China continental donde se abordan distintos aspectos que influyen sobre la renuencia a hablar. Estos análisis ni siquiera tienen por qué centrarse concretamente en la resistencia a hablar, así ocurre con las investigaciones de Muñoz Torres (2010a, 2010b) sobre la ansiedad, que no es más que una de las dimensiones que influyen sobre el fenómeno de la renuencia. Para un tratamiento más global de este fenómeno con estudiantes de Asia Oriental, véase Mendoza (2016, 2017, 2021).

2 No recogemos aquí una nómina exhaustiva.
} 
Loh y Teo, 2017; Mantiri, 2013; Sugahara y Boland, 2010) como por el entorno que rodea al individuo (Kolb, 1984). De modo que los estilos de enseñanza, el ambiente de aprendizaje, así como el entrenamiento recibido en las distintas etapas del sistema educativo ejercerán su influencia sobre dichos estilos (Buyse y Morera, 2016). Y precisamente debido a este hecho, tal como indicaron Davidman (1981), Reid (1987) o Kennedy (2002), los estilos de aprendizaje, aunque estables, no son fijos, sino que presentan la posibilidad de cambiar o de ser modificados. Algunos trabajos, como los de Barron y Arcodia (2002), Loh y Teo (2017), Volet y Renshaw (1996) y Xia (2009), ya dejaron constancia de este hecho. En ellos se percibían modificaciones en los estilos de aprendizaje de los estudiantes tras disfrutar de una estancia en otro país o bien adaptaciones de estos al integrarse en un ambiente académico donde el estilo dominante en el grupo o aquel requerido por el entorno de aprendizaje era distinto al acostumbrado.

Ahora bien, el concepto de estilos de aprendizaje no es menos complejo que el de la renuencia a hablar, por lo que el número de definiciones y clasificaciones existentes es abundante. De manera general, los estilos de aprendizaje podrían definirse como el modo que cada individuo tiene de acercarse al aprendizaje, como el modelo que describe la forma preferida en la que un sujeto percibe, responde e interactúa con los ambientes de aprendizaje (Dörnyei, 2005). En este trabajo nos centraremos en la definición ofrecida por Honey y Mumford (1986), en cuyo modelo se basa el cuestionario que emplearemos más adelante. Según estos autores, los estilos de aprendizaje pueden ser entendidos como una descripción de las actitudes y el comportamiento que determina la forma de aprender preferida por un individuo. Honey y Mumford parten de la teoría del aprendizaje a partir de la experiencia de Kolb (1984), pero, basándose en su definición de los estilos, elaboran un cuestionario, el Learning Styles Questionnaire, no enfocado en cuestiones directamente relacionadas con el aprendizaje, sino orientado a conocer las tendencias generales de comportamiento de los informantes. Para estos autores, el aprendizaje se concebiría como un proceso cíclico formado por cuatro etapas: tener una experiencia o experimentar, revisar la experiencia o reflexionar, sacar conclusiones de la experiencia o elaborar hipótesis y planificar los pasos siguientes o aplicar dichas hipótesis. De estas etapas o fases surgen los cuatro estilos diferenciados por Honey y Mumford (1986): activo, reflexivo, teórico y pragmático. A este respecto, aunque lo deseable sería que todos empleáramos de forma equilibrada todas estas virtualidades, lo cierto es que solemos tener más desarrolladas unas capacidades que otras y normalmente manifestamos una mayor preferencia por unos estilos de aprendizaje frente a otros.

El cuestionario de Honey y Mumford, que fue aplicado sobre todo al ámbito empresarial, sería adaptado al ámbito académico por Alonso. De esta forma surgiría el Cuestionario Honey-Alonso de Estilos de Aprendizaje, más conocido como CHAEA (Alonso et alii, 1995). Siguiendo las definiciones dadas por estos autores, resumimos las características principales de cada estilo: 
a) Activo.- Les encanta la actividad, las nuevas experiencias y los desafíos. Son grupales y de mente abierta, arriesgados, espontáneos, animadores, descubridores e improvisadores. Les aburren los largos plazos.

b) Reflexivo.- Les gusta observar las experiencias desde diferentes perspectivas, por eso escuchan y observan a los demás para apropiarse de una situación y tienen en cuenta las distintas alternativas antes de actuar. Para alcanzar una conclusión, reúnen datos y los analizan detenidamente. Son receptivos, analíticos, concienzudos, ponderados.

c) Teórico.- Les gusta analizar, sintetizar y enfocar los problemas por etapas lógicas. Integran observaciones y hechos en teorías coherentes y complejas. Huyen de la subjetividad y organizan su sistema de pensamiento de acuerdo a principios, modelos y teorías. Son objetivos, críticos, lógicos, estructurados, metódicos.

d) Pragmático.- Les gusta aplicar las ideas que les atraen de forma práctica y experimentarlas aprovechando la primera oportunidad que se les presente. Les impacienta la teorización. Toman decisiones y resuelven problemas de forma sensata. Si algo funciona, es bueno para ellos. Son prácticos, eficaces, directos, experimentadores, realistas.

Como expusimos más arriba, son numerosos los investigadores que reconocen la influencia que la cultura de un individuo ejerce sobre sus preferencias de aprendizaje. De ahí que en distintos trabajos se hayan buscado relaciones entre determinados valores culturales arraigados en una comunidad y las preferencias de aprendizaje de sus miembros. En este sentido, las dimensiones culturales de Hofstede han sido muy utilizadas (Hofstede et alii, 2010). Basándose en ellas, se ha establecido, por un lado, una relación más o menos generalizada entre aquellas culturas colectivistas y de alta o moderada distancia jerárquica y los estilos de aprendizaje reflexivo y teórico ${ }^{3}$, frente aquellas culturas más individualistas y de baja distancia jerárquica, por otro, y los estilos de aprendizaje activo y pragmático (Auyeung y Sands, 1996; Aziz et alii, 2013; Bhatnagar y Sinha, 2018; Charlesworth, 2008; Joy y Kolb, 2008; Martínez, 2012; Sugahara y Boland, 2010; Srivoranart, 2015; Syed, 2006). En esta línea, Taiwán, al igual que otros países de mayoría poblacional china o de bases culturales confucianistas, presenta una moderada distancia jerárquica y un colectivismo elevado (Hofstede et alii, 2010). De modo que, de acuerdo con lo que acabamos de exponer y tal como hallaron Auyeung y Sands (1996), sería esperable que la mayoría de los estudiantes taiwaneses presentaran una preferencia alta por los estilos de aprendizaje reflexivo y teórico, en detrimento de los otros dos estilos de aprendizaje. Es evidente que, de acuerdo con las descripciones previas, los estilos reflexivo y teórico se asocian a comportamientos menos arriesgados, directos o experimentadores y, en cambio, más calmados, receptivos o analíticos; por lo que puede inferirse una menor tendencia a la

\footnotetext{
${ }^{3}$ Algunos autores, como Joy y Kolb (2008), aunque no aluden a los estilos de Honey y Mumford (1986), observan en estos colectivos una mayor preferencia por el pensamiento abstracto, rasgo central del estilo teórico.
} 
participación en dinámicas de trabajo activas o comunicativas y una mayor facilidad para caer en la renuencia a hablar en clase.

No obstante, a pesar de que muchos autores apuntan en la dirección que hemos señalado, no podemos perder de vista que existen investigaciones cuyos resultados difieren de esta tendencia. Sirva de ejemplo el trabajo de Mitsis y Foley (2004), en el que se hallaron asociaciones positivas entre los estudiantes de culturas colectivistas y el estilo pragmático. De igual modo, años antes, Chou et alii (1999), en un análisis de los estilos de aprendizaje de los alumnos taiwaneses de Contabilidad, encontraron una mayor preferencia por el estilo pragmático frente a una preferencia moderada por los estilos reflexivo y teórico, y baja por el estilo activo.

Tras esta somera revisión de la bibliografía, cabe destacar que, a pesar de la posible relación existente entre el fenómeno de la renuencia a hablar en clase y los estilos de aprendizaje del alumnado, desconocemos que se hayan llevado a cabo estudios en esta dirección dentro del área del español como lengua extranjera en los países de Asia Oriental. Es más, incluso desconocemos la existencia de investigaciones sobre los estilos de aprendizaje de los estudiantes taiwaneses de ELE ${ }^{4}$. Es cierto que existen textos relacionados con estrategias of factores diversos que pueden incidir sobre el aprendizaje de los alumnos taiwaneses, pero no hemos encontrado bibliografía donde los estilos de aprendizaje, en tanto que indicadores estables y medibles, sean identificados en grupos de estudiantes empleando instrumentos de evaluación. Ampliando nuestro radio a todo el ámbito sinohablante, ha sido Feng (2019) quien recientemente ha llevado a cabo investigaciones de diagnóstico, con aprendientes de China continental, en las que ha empleado el Cuestionario Honey-Alonso de Estilos de Aprendizaje. En consecuencia, con este trabajo pretendemos arrojar algo más de luz sobre estos aspectos.

\section{Objetivos}

- Conocer el grado de renuencia hablar y sus causas entre los estudiantes taiwaneses de ELE de la muestra seleccionada.

- Conocer las preferencias de aprendizaje de estos estudiantes.

- Saber si existe alguna relación entre el fenómeno de la renuencia a hablar en clase y los distintos estilos de aprendizaje.

\section{Procedimiento}

Presentamos aquí un estudio cuantitativo cuya primera parte es descriptiva, ya que se busca describir tanto el fenómeno de la renuencia como las preferencias de aprendizaje del alumnado; mientras que la segunda parte es correlacional, pues se buscan relaciones entre los distintos estilos de aprendizaje y el primer fenómeno

\footnotetext{
${ }^{4}$ Cabe destacar que las investigaciones sobre estilos de aprendizaje llevadas a cabo en el área de la enseñanzaaprendizaje del inglés en Taiwán son diversas, pero emplean cuestionarios muy variados para clasificar estos estilos. A excepción del texto de Auyeung y Sands (1996) y el de Chou et alii (1999), no conocemos otros trabajos que empleen la clasificación de Honey y Mumford, por lo que la comparación con los estilos del CHAEA se hace difícil.
} 
analizado. Para medir y analizar la renuencia a hablar empleamos la Reticence Scale-12 (RS-12) (Kelly et alii, 2010), versión reducida de la anterior RS-24 (Keaten et alii, 1997), cuestionario que ha demostrado una sobrada validez y fiabilidad en trabajos de muy diversos investigadores (Baktash y Chalak, 2016; Chalak y Baktash, 2015; Chowdhury, 2016; Mendoza, 2017; O'Connell, 2010; Soo y Goh, 2013). En nuestro caso reveló una alta consistencia interna con un alfa de Cronbach de 0,914. La RS-12 mide la renuencia a comunicarse en relación con seis dimensiones vinculadas a tres componentes básicos: conductual, cognitivo y afectivo (Keaten y Kelly, 2000). Estas seis dimensiones son: ansiedad, conocimiento, tiempo, organización, forma de hablar y memoria. Cada dimensión se mide mediante dos afirmaciones que han de ser valoradas según una escala Likert de cinco puntos. Puesto que la puntuación máxima que puede obtenerse en el cuestionario es de 60 puntos, estimamos que presenta una renuencia considerable aquel encuestado que obtenga una puntuación igual o superior a 30 (Mendoza, 2017; Soo y Goh, 2013).

Por otro lado, para estudiar los estilos de aprendizaje empleamos el ya citado Cuestionario Honey-Alonso de Estilos de Aprendizaje (CHAEA) (Alonso et alii, 1995). Este cuestionario ha sido ampliamente utilizado tanto en España como en Hispanoamérica con estudiantes universitarios, así como, en los últimos años, también aplicado en el área de la enseñanza-aprendizaje del ELE (Feng, 2019; Luengo-Cervera, 2015; Martínez, 2012; Morera, 2016: Srivoranart, 2015; Zolnay, 2013). El CHAEA consta de 80 afirmaciones de respuesta dicotómica, 20 por cada estilo, y en él solo se computan las respuestas positivas. Para medir el grado de preferencia por cada estilo, utilizaremos la adaptación del baremo de Honey-Alonso, realizada por Feng (2019), para alumnos chinos estudiantes de español ${ }^{5}$. En este se distinguen tres grados: bajo, moderado y alto frente a los cinco del original:

Tabla 1. Adaptación del baremo de Honey-Alonso para alumnos chinos (Feng, 2019)

\begin{tabular}{llll}
\hline Estilos de aprendizaje & \multicolumn{3}{c}{ Preferencias } \\
\hline & Baja & Moderada & Alta \\
Activo & $0-9$ & $10-13$ & $14-20$ \\
Reflexivo & $0-14$ & $15-17$ & $18-20$ \\
Teórico & $0-12$ & $13-15$ & $16-20$ \\
Pragmático & $0-11$ & $12-14$ & $15-20$ \\
\hline
\end{tabular}

Ambos cuestionarios fueron traducidos a la lengua de los estudiantes y estos fueron debidamente informados acerca de la finalidad de las encuestas. Todos ellos dieron su consentimiento para participar en el proyecto. La muestra está formada por 240 alumnos del Departamento de Lengua y Literatura Españolas de la Universidad

\footnotetext{
${ }^{5}$ Baremo actualizado y que se ajusta mejor al perfil del estudiante oriental, ya que el de Alonso et alii (1995) fue establecido a partir de encuestas realizadas a universitarios españoles.
} 
Providence ${ }^{6}$, de segundo, tercero y cuarto curso de grado ${ }^{7}$. Se buscó una distribución similar por cursos, es decir, 80 informantes por cada curso. Los estudiantes del primer curso quedaron excluidos para evitar que el periodo silente pudiera afectar a los resultados obtenidos en la RS-12. De ellos, 72 eran hombres y 168, mujeres ${ }^{8}$. Tan solo el $13 \%$ de la muestra había disfrutado de una estancia en el extranjero ${ }^{9}$ (todos ellos estudiantes de cuarto curso). Por otro lado, el 8\% afirmó estar en posesión de algún diploma DELE, en su mayoría de nivel B1. En cuanto a la docencia recibida, el 88\% había tenido clases al menos con un profesor nativo, si bien, solo los estudiantes de tercero y cuarto, es decir, el 75\% había disfrutado de clases de conversación con un profesor de esta índole. Los cuestionarios fueron distribuidos a lo largo del curso 2019-2020. El análisis de estos y las correlaciones estadísticas se realizaron utilizando el paquete SPSS25 para Windows.

\section{Resultados}

Atendamos, en primer lugar, a los resultados de la RS-12, con los que conoceremos la presencia que el fenómeno de la renuencia a hablar tiene entre los alumnos encuestados. En un acercamiento inicial, los estadísticos descriptivos básicos de los totales arrojan una media de 37,5 puntos, una mediana de 36 y una moda de 34 , lo que apunta hacia un conjunto de valores que parece situarse de manera mayoritaria por encima de los 30 puntos. Este hecho queda corroborado por el número de estudiantes que puede ser catalogado como reacio a hablar en clase: un total de 180 (75\%) frente a los 60 (25\%) cuya renuencia comunicativa es baja. La distribución de los estudiantes reacios a hablar por curso académico sería la siguiente: segundo curso, 57 estudiantes; tercero, 64; cuarto, 59. De modo que el fenómeno de la renuencia a hablar tiene una importante representatividad en todos los cursos de este estudio, con cifras superiores al 70\% de los encuestados de cada uno de los grupos. De acuerdo con estos datos, existe una considerable mayoría de informantes para los que hablar en la clase de español supone bien un problema, bien una dificultad adicional dentro del propio proceso de aprendizaje. Estos resultados son semejantes a los obtenidos por investigaciones similares llevadas a cabo en otros países asiáticos. En ellas se identificó a una mayoría de alumnos renuentes a hablar en las clases de inglés (Baktash y Chalak, 2016; Chalak y Baktash, 2015; Musytarif, 2015; Soo y Goh 2013) o en las de español como lengua extranjera (Mendoza, 2017). Una vez que hemos identificado al 75\% de los encuestados como renuentes a hablar, nos interesa conocer en qué dimensiones o afirmaciones del

\footnotetext{
${ }^{6}$ Uno de los cuatro departamentos de español que existen en la isla.

7 Dentro del contexto taiwanés de aprendizaje de ELE, como hemos indicado, nuestros encuestados pertenecen a la enseñanza reglada universitaria, concretamente, de carácter privado. Normalmente estos estudiantes llegan a la carrera sin conocimientos sobre el español, lengua que estudiarán a partir de un currículo que divide la enseñanza por destrezas dando lugar a distintas asignaturas (Lectura y Composición, Audición y Conversación; aunque en los cursos superiores estas últimas aparecen unidas) más otra asignatura de gramática. En los primeros cursos suelen tener profesores taiwaneses, y es a partir de tercero cuando empiezan a tener profesores nativos, sobre todo en las asignaturas de conversación.

8 Este desequilibrio se ajusta a la realidad del estudiantado, con una considerable mayoría de alumnas.

${ }^{9} \mathrm{El} \mathrm{75 \%}$ de las estancias fueron por prácticas laborales, con una duración bimestral, bien en España o en un país hispanoamericano. El 25\% restante fueron estancias por estudios, con destinos similares y una duración variable entre los 2 y los 6 meses.
} 
cuestionario se apoya este fenómeno. Con ese objetivo hemos creado la siguiente tabla donde se recogen tanto la frecuencia $(f)$ como el porcentaje (\%) de estudiantes que se muestran «totalmente de acuerdo / de acuerdo», «indeciso» o «totalmente en desacuerdo / en desacuerdo» ${ }^{10}$ con cada una de las afirmaciones ${ }^{11}$ :

Tabla 2. Respuestas de los encuestados a las afirmaciones de la encuesta

\begin{tabular}{|c|c|c|c|c|c|c|}
\hline \multirow{2}{*}{ Dimensiones y afirmaciones } & \multicolumn{2}{|c|}{ TDA / DA ${ }^{12}$} & \multicolumn{2}{|c|}{ Indeciso } & \multicolumn{2}{|c|}{ TDS/ DS } \\
\hline & $\mathrm{f}$ & $\%$ & $\mathrm{f}$ & $\%$ & $\mathrm{f}$ & $\%$ \\
\hline \multicolumn{7}{|l|}{ 1. Ansiedad } \\
\hline a) Estoy nervioso & 163 & 67,9 & 15 & 6,2 & 62 & 25,8 \\
\hline b) Me siento tenso & 130 & 54,1 & 36 & 15 & 74 & 30,8 \\
\hline \multicolumn{7}{|l|}{ 2. Forma de hablar } \\
\hline a) Confundo las palabras & 135 & 56,2 & 32 & 13,3 & 73 & 30,4 \\
\hline b) Me trabo & 118 & 49,1 & 32 & 13,3 & 90 & 37,5 \\
\hline \multicolumn{7}{|l|}{ 3. Memoria } \\
\hline a) Me olvido de lo que quiero decir & 118 & 49,1 & 27 & 11,2 & 95 & 39,5 \\
\hline $\begin{array}{l}\text { b) Pierdo de vista lo que quiero } \\
\text { decir }\end{array}$ & 65 & 27 & 47 & 19,5 & 128 & 53,3 \\
\hline \multicolumn{7}{|l|}{ 4. Organización } \\
\hline a) Mis ideas están desordenadas & 83 & 34,5 & 54 & 22,5 & 103 & 42,9 \\
\hline b) Mis ideas están mezcladas & 66 & 27,5 & 41 & 17 & 133 & 55,4 \\
\hline \multicolumn{7}{|l|}{ 5. Tiempo } \\
\hline $\begin{array}{l}\text { a) Necesito demasiado tiempo para } \\
\text { decir lo que quiero }\end{array}$ & 137 & 57 & 30 & 12,5 & 73 & 30,4 \\
\hline $\begin{array}{l}\text { b) Dudo demasiado para decir lo } \\
\text { que quiero }\end{array}$ & 92 & 38,3 & 41 & 17 & 107 & 44,5 \\
\hline \multicolumn{7}{|l|}{ 6. Conocimiento } \\
\hline $\begin{array}{l}\text { a) Desconozco el tema sobre el que } \\
\text { hay que hablar }\end{array}$ & 139 & 57,9 & 34 & 14,1 & 67 & 27,9 \\
\hline $\begin{array}{l}\text { b) No estoy familiarizado con el } \\
\text { tema }\end{array}$ & 69 & 28,7 & 47 & 19,5 & 124 & 51,6 \\
\hline
\end{tabular}

Centrémonos ahora en las respuestas indicadoras de renuencia, es decir, en aquellas donde los informantes se muestran de acuerdo con las afirmaciones de la RS12. Dos son las dimensiones que destacan en conjunto sobre las demás: en primer lugar, la dimensión «ansiedad» seguida de la dimensión «forma de hablar». La dimensión «ansiedad» es la que más sobresale sobre el resto, ya que el 67,9\% de los encuestados considera que está nervioso cuando habla y el $54,1 \%$ se siente tenso al hablar en clase. En segundo lugar, es la dimensión «forma de hablar» la que, teniendo en cuenta los

\footnotetext{
10 La agrupación de los ítems que guardan relación entre sí se debe a cuestiones de espacio.

${ }^{11}$ Las afirmaciones se refieren al momento en el que el alumno habla español en clase. A cada afirmación de la tabla habría que añadirle: «(...) cuando hablo español en clase». Así aparecía explicado en la encuesta que se repartió.

12 TDA «totalmente de acuerdo»; DA «de acuerdo»; TDS «totalmente en desacuerdo»; DS «en desacuerdo».
} 
resultados de sus dos afirmaciones, se sitúa en segunda posición. En ella el 56,2\% considera que cuando habla confunde las palabras y el 49,1\% estima que se traba al intervenir en clase. En el extremo opuesto se situaría la dimensión «organización», con cuyas afirmaciones se muestra de acuerdo el menor número de estudiantes. En las tres dimensiones restantes, encontramos, curiosamente, una afirmación que destaca de manera clara sobre la otra. Así en la dimensión «conocimiento», el 57,9\% de los alumnos afirma que no habla porque desconoce el tema propuesto. Del mismo modo, en la dimensión «tiempo», el 57\% estima que necesita demasiado tiempo para decir lo que quiere, e igualmente, en la dimensión «memoria», un 49,1\% afirma que se olvida de lo que quiere decir.

Atendamos ahora a los resultados obtenidos en el CHAEA. Comencemos conociendo las cifras arrojadas por las medidas de tendencia central:

Tabla 3. Medidas de tendencia central sobre los totales del CHAEA

\begin{tabular}{lllll}
\hline & Activo & Reflexivo & Teórico & Pragmático \\
\hline Media & 12,31 & 15,19 & 13,53 & 13,32 \\
Mediana & 12 & 16 & 14 & 13 \\
Moda & 12 & 18 & 15 & 12 \\
\hline
\end{tabular}

Prestando atención a los valores previos, puede observarse cómo las medias de todos los estilos de aprendizaje se sitúan dentro de la franja de preferencia moderada. Lo mismo ocurre con las modas de los estilos activo, teórico y pragmático. En cambio, el valor que más se repite para el estilo reflexivo pertenece a la franja de preferencia alta (18); sin embargo, esa media de 15,19 parece apuntar hacia la existencia de bastantes puntuaciones comprendidas dentro de la franja de preferencia baja.

Veamos a continuación la distribución del número de encuestados (frecuencia) y sus correspondientes porcentajes, de acuerdo con sus preferencias por cada estilo de aprendizaje:

Tabla 4. Preferencias para cada estilo de aprendizaje

\begin{tabular}{|c|c|c|c|c|c|c|c|c|}
\hline \multirow[t]{3}{*}{ Preferencias } & \multicolumn{6}{|c|}{ Estilos de aprendizaje } & \multirow{2}{*}{\multicolumn{2}{|c|}{ Pragmático }} \\
\hline & \multicolumn{2}{|c|}{ Activo } & \multicolumn{2}{|c|}{ Reflexivo } & \multicolumn{2}{|c|}{ Teórico } & & \\
\hline & $f$ & $\%$ & $\mathrm{f}$ & $\%$ & $\mathrm{f}$ & $\%$ & $\mathrm{f}$ & $\%$ \\
\hline Alta & 82 & 34,1 & 63 & 26,2 & 60 & 25 & 83 & 34,5 \\
\hline Moderada & 105 & 43,7 & 82 & 34,1 & 104 & 43,3 & 105 & 43,7 \\
\hline Ваја & 53 & 22 & 95 & 39,5 & 76 & 31,6 & 37 & 21,6 \\
\hline Total & 240 & 100,0 & 240 & 100,0 & 240 & 100,0 & 240 & 100,0 \\
\hline
\end{tabular}

Como puede apreciarse en la tabla, la mayoría de los estudiantes encuestados presenta una preferencia moderada por el estilo activo, teórico o pragmático. De hecho, el número de alumnos con preferencia moderada por uno de estos tres estilos es casi el mismo: 105 (43,7\%) para los estilos activo y pragmático y $104(43,3 \%)$ para el estilo teórico. Es únicamente en el estilo reflexivo donde la preferencia baja $(95 / 39,5 \%)$ es 
superior a las otras dos franjas, rozando el $40 \%$ del total de la muestra. Si nos fijamos en las preferencias altas de los informantes, cabe destacar que los estilos que presentan un mayor porcentaje en esta franja son los estilos activo y pragmático, ambos por encima del 34\% del total (34,1\%/34,5\%). Mientras que, contra lo esperado, los estilos reflexivo y teórico son los que presentan un menor porcentaje de preferencia alta entre los estudiantes, situándose en torno al 25\% (26,2\%/25\%). Del mismo modo, y también en contra de lo que sería esperable, los dos estilos que en los que los alumnos presentaron un menor porcentaje de preferencia baja fueron el activo y el pragmático $(22 \% / 21,6 \%)$, frente a los mayores resultados en esta franja de los estilos reflexivo y teórico $(39,5 \% / 31,6 \%)$.

Una vez expuestos los resultados de la RS-12 y del CHAEA, centrémonos en averiguar si existe alguna asociación entre los resultados obtenidos en la escala de renuencia y aquellos obtenidos en el Cuestionario Honey-Alonso de Estilos de Aprendizaje. Con este fin hemos utilizado el coeficiente de correlación de Spearman ${ }^{13}$, que nos permitirá medir la magnitud y dirección de asociación entre estas variables.

Tabla 5. Rho de Spearman

\begin{tabular}{|c|c|c|c|c|}
\hline & Activo & Reflexivo & Teórico & Pragmático \\
\hline $\begin{array}{l}\text { Coeficiente de } \\
\text { correlación }\end{array}$ & $-0,222^{*}$ & 0,049 & 0,091 & $-0,217^{*}$ \\
\hline $\begin{array}{l}\text { Significación } \\
\text { bilateral }\end{array}$ & $0,003^{*}$ & 0,518 & 0,235 & $0,004^{*}$ \\
\hline
\end{tabular}

Como muestra la tabla previa, la correlación de Spearman muestra correlaciones negativas y significativas entre la renuencia a hablar y los estilos activo (rho $=-0,222$ [p $<0,05]$ ) y pragmático (rho $=-0,217[\mathrm{p}<0,05]$ ). Si bien estas correlaciones son bajas, ya que el coeficiente supera levemente el valor 0,2 . El resto de los estilos no presentan correlaciones significativas.

\section{Discusión}

De acuerdo con los datos previos, en la muestra seleccionada se aprecia una vez más la existencia de un importante número de estudiantes asiáticos (más del 75\%) reacios a participar en las clases de lenguas extranjeras, concretamente en las clases de ELE. Entre nuestros informantes, las razones que ejercen un mayor peso como causantes de este fenómeno son aquellas vinculadas a las dimensiones «ansiedad» y «forma de hablar», tal como ocurría en las muestras de Baktash y Chalak (2016), Chalak y Baktash (2015), Musytarif (2015) y Soo y Goh (2013). La ansiedad se erige como la dimensión que más bloquea a nuestros aprendientes a la hora de intervenir en el aula, poniendo nuevamente de manifiesto la importancia de los factores afectivos en el aprendizaje de lenguas extranjeras. No en vano, como expusieron Arnold y Brown

\footnotetext{
13 Empleamos la prueba no paramétrica de Spearman, ya que la prueba de Kolmogorov-Smirnov, aplicada a las variables en juego, reveló la no normalidad de estas.
} 
(2000), «la ansiedad es probablemente el factor afectivo que obstaculiza con más fuerza el proceso de aprendizaje», estando asociada a elementos negativos como «el desasosiego, la frustración, la inseguridad, el miedo o la tensión». Los métodos de enseñanza más centrados en la comunicación unidos a la inseguridad que genera el tener que comunicarse con un vehículo lingüístico inestable, como es una lengua ajena a la propia, han aumentado las situaciones que provocan ansiedad en clase. La relevancia de la dimensión ansiedad en nuestra muestra coincide también con la importancia otorgada a esta dimensión en la revisión del constructo de la renuencia, llevada a cabo por Keaten y Kelly (2000) sobre la original concepción de Phillips (1965). Junto a las dimensiones «ansiedad» y «forma de hablar», entre las respuestas de nuestros encuestados destacaban otras tres afirmaciones pertenecientes a los apartados «memoria», «tiempo» y «conocimiento». De modo que nuestros alumnos ven limitada, además, su capacidad de comunicarse ya que, desde su punto de vista, olvidan lo que quieren decir, no conocen bastante el tema del que tienen que hablar o necesitan más tiempo para expresarse. Elementos que ya aparecen, en mayor o menor medida, en otras investigaciones. Por ejemplo, las demandas del alumnado asiático respecto al escaso tiempo concedido para organizar y articular su discurso han sido recogidas por autores como Bao (2014), Chang (2011), Liu y Littlewood (1997) o Mendoza (2017), entre otros. En los trabajos de Chalak y Baktash (2015), Baktash y Chalak (2016), y Soo y Goh (2013), más de un 40\% de sus informantes afirman olvidar lo que quieren decir cuando les toca hablar en clase. De igual forma, el desconocimiento del tema propuesto y su influencia sobre la resistencia a hablar ya aparece mencionado en algunos textos como Liu (2005), Mendoza (2016) o Bao (2014). En definitiva, esta diversidad de causas identificada en nuestra encuesta pone claramente de manifiesto la ya citada complejidad del fenómeno analizado así como su perfil poliédrico.

En lo relativo a los estilos de aprendizaje, los datos arrojados por el CHAEA ofrecen una imagen de un estudiantado con preferencias predominantemente moderadas en los estilos activo, teórico y pragmático, y con una preferencia mayoritariamente baja por el estilo reflexivo. Estos resultados coinciden en gran medida con aquellos obtenidos recientemente por Feng (2019) y Feng et alii (2019) con estudiantes chinos de español. El hecho de que el estilo reflexivo se desmarque de los otros tres estilos y presente una considerable preferencia baja entre el alumnado hace que nuestros resultados difieran de aquellos obtenidos por Auyeung y Sands (1996), Aziz et alii (2013), Martínez (2012), Srivoranart (2015), Syed (2006) y Sugahara y Boland (2010). En ellos se identificó una clara preferencia por el estilo reflexivo o teórico entre grupos de estudiantes de distintos países de Asia con valores culturales similares. Nuestros resultados tampoco sirven para corroborar la teoría de Bhatnagar y Sinha (2018), según la cual el número de alumnos con estilo reflexivo dominante es mayor en aquellas sociedades colectivistas con una considerable distancia jerárquica. Por otro lado, también en contra de lo esperado, las preferencias altas registran mayores porcentajes en los estilos activo y pragmático que en los estilos tradicionalmente asociados a nuestro perfil de 
estudiante $^{14}$. Estos resultados podrían relacionarse parcialmente con aquella asociación positiva hallada por Mitsis y Foley (2004) entre los estudiantes de sociedades colectivistas y el estilo de aprendizaje pragmático. No obstante, también podrían relacionarse con una modificación de sus preferencias originales tal como ya apuntaron los estudios de Loh y Teo (2017), Barron y Arcodia (2002), y Volet y Renshaw (1996). Y aunque en estas últimas investigaciones los estudiantes presentaban cambios en sus estilos de aprendizaje tras una estancia en el extranjero, también existen otros trabajos como los de Kennedy (2002) o Finch (2008) donde los estudiantes adoptaron estilos más activos en su país de origen tras experimentar un modo de enseñanza más comunicativo y centrado en la figura del alumno. De este modo, no sería de extrañar que se hubiera producido cierta modificación en sus preferencias tras una práctica más dinámica e interactiva en las clases de español con nativos.

Por último, cabe mencionar la ausencia de correlaciones significativas entre los estilos reflexivo y teórico y la renuencia a hablar en clase, así como la existencia de una correlación negativa y baja entre este fenómeno y los estilos activo y pragmático. No podemos afirmar, tal como sería esperable de acuerdo con la bibliografía, que exista una relación entre los estilos reflexivo o teórico y la renuencia a hablar en clase. Es decir, no puede afirmarse que cuanto mayor sea la preferencia por los estilos reflexivo o teórico, más se acentúe la resistencia a comunicarse. En cambio, la prueba estadística empleada parece indicar que conforme el estilo activo o pragmático aumenta como preferencia, la renuencia a hablar en clase disminuye y viceversa.

\section{Conclusiones}

En las páginas previas, hemos medido tanto la renuencia comunicativa como las preferencias de aprendizaje de un conjunto de estudiantes taiwaneses del grado en Lengua y Literatura Españolas. Posteriormente se ha analizado si existía alguna correlación entre los resultados de la renuencia a hablar y las distintas preferencias de aprendizaje. La muestra estudiada presenta una clara mayoría que es renuente a hablar en las clases de español. Las principales razones de esta resistencia son las que conforman la dimensión «ansiedad», seguidas de cerca por aquellas causas que componen la dimensión «forma de hablar». Las dimensiones «conocimiento», «tiempo» $\mathrm{y}$ «memoria» también aportan razones para justificar este fenómeno, lo que confirma su complejidad y su carácter multidimensional. La toma de acciones concretas, por parte de los profesores, que sirvan para desarrollar la confianza, aumentar la autopercepción, promover un clima agradable de interacción, así como cierta modificación en la gestión del tiempo otorgado al alumnado para preparar sus intervenciones puede mitigar este fenómeno y mejorar el índice de participación en el aula. Por otro lado, los resultados de las preferencias de aprendizaje apuntan en una dirección diferente a la esperada. Nuestra muestra no presenta elevadas preferencias por el estilo reflexivo o teórico, como habían descrito algunos autores para el perfil del alumnado encuestado. Es más, fue el estilo reflexivo el que obtuvo un porcentaje más

14 También ocurre en Feng (2019). 
elevado de preferencia baja. Tampoco puede identificarse en la muestra un claro estilo dominante: el predominio de preferencias moderadas impide este hecho. Otro aspecto que resulta interesante es que los estilos activo y pragmático registraran un mayor porcentaje de preferencia alta que los otros dos estilos restantes, pudiendo indicar un cambio en las preferencias de aprendizaje tradicionalmente asociadas a los miembros de sociedades colectivistas con alta o moderada distancia jerárquica. Esta modificación podría estar asociada a su exposición, sobre todo en las clases con profesores nativos, a nuevos enfoques didácticos más activos y comunicativos, en los que pesaría más el carácter práctico de la lengua que el teórico. Este posible cambio en curso vendría a corroborar la afirmación de Littlewood (2000), quien consideraba que en estas sociedades el predominio de los estilos reflexivo y teórico podría deberse más al entorno de aprendizaje y a las prácticas docentes tradicionales que a características intrínsecas del alumnado.

Por último, la ausencia de correlación significativa entre los estilos reflexivo y teórico y el nivel de renuencia a hablar pone de manifiesto que sobre este fenómeno influyen más otros factores que estos dos estilos de aprendizaje. En cambio, la correlación negativa y significativa establecida entre la renuencia y los estilos activo y pragmático apunta hacia una influencia positiva de estos dos estilos sobre la participación en el aula.

\section{Referencias bibliográficas}

ALONSO, C. M. et alii (1995). Los Estilos de Aprendizaje: Procedimientos de diagnóstico y mejora. Bilbao: Ediciones Mensajero.

ARNOLD, J. y BROWN, H. D. (2000). Mapa del terreno. En J. ARNOLD (ed.). La dimensión afectiva en el aprendizaje de idiomas. Cambridge. Cambridge University Press, 1941.

ARNOLD, J. y FONSECA, M. C. (2004). Reflexiones sobre aspectos del desarrollo de la competencia comunicativa oral en el aula de español como segunda lengua. En F. LORENZO y S. RUHSTALLER (coords.). La competencia lingüística y comunicativa en el aprendizaje de español como lengua extranjera. Sevilla. Edinumen, 45-60.

AUYEUNG, P. y SANDS, J. S. (1996). A cross-cultural study of the learning style of accounting students. Accounting and Finance, 36, 261-274.

AZIZ, Z. et alii (2013). Learning Style Preferences of Pharmacy Students. The European Journal of Social \& Behavioural Sciences, 4(1), 819-835.

BAO, D. (2014). Understanding Silence and Reticence. Ways of Participating in Second Language Acquisition. Nueva York: Bloomsbury.

BAKTASH, F. y CHALAK A. (2016). The Relationship between Reticence and Personality Types in Iranian University EFL Classrooms. Theory and Practice in Language Studies, 6(5), 1000-1005.

BARRON, P. y ARCODIA, C. (2002). Linking Learning Style Preferences and Ethnicity: International Students Studying Hospitality and Tourism Management in Australia. Journal of Hospitality, Leisure, Sport \& Tourism Education, 1, 15-27. 
BHATNAGAR, T. y SINHA, V. (2018). Learning Styles: A Comparison between Indian and German Business Students. Journal of International Students, 8(1), 473-487.

BUYSE, K. y MORERA, I. (2016). Perception of educational culture and learning styles in language learning: the Romanian case. Revista Nebrija de Lingüística Aplicada, 21, $1-14$

CHANG, F. Y. (2011). The Causes of Learners' Reticence and Passivity in English Classrooms in Taiwan. The Journal of Asia TEFL, 8(1), 1-22.

CHALAK, A. y BAKTASH, F. (2015). An Investigation on Students' Reticence in Iranian University EFL Classrooms. International Scholarly and Scientific Research \& Innovation, 9(8), 2370-2373.

CHARLESWORTH, Z. (2008). Learning styles across cultures: suggestions for educators. Education \& Training, 50(2), 115-127.

CHOU, T. et alii (1999). The effects of learning tendencies, learning approaches and learning habits on academic achievement: Evidence from Taiwanese college accounting students. En K. MARTIN, N. STANLEY y N. DAVISON (eds.). Teaching in the Disciplines/Learning in Context, Australia. Perth. University of Western Australia, 76-83.

CHOWDHURY, R. F. (2016). Reasons of reticence in speaking English among the freshmen in Port City International University; Chittagong, Bangladesh: a case study. EKL Asia. Pacific Journal of Social Sciences, 2(3), 1-22.

CORTAZZI, M. y JIN, L. (1996). Cultures of learning: language classrooms in China. En H. COLEMAN (ed.). Society and the Language Classroom. Cambridge. Cambridge University Press, 169-206.

DAVIDMAN, L. (1981). Learning style: The myth, the panacea, the wisdom. The Phi Delta Kappan, 62, 641-645.

DÖRNEY, Z. (2005). The Psychology of the Language Learner. Individual differences in Second Language Acquisition. Nueva York: Routledge.

FENG, Y. (2019). Aproximación cualitativa sobre la aplicación del CHAEA en los estudiantes chinos que aprenden español como Lengua Extranjera. Revista de Estilos de Aprendizaje, 12(24), 17-27.

FENG, Y. et alii (2019). Relationship between Learning Styles, Learning Strategies and Academic Performance of Chinese Students Who Learn Spanish as a Foreign Language. The Asia Pacific Education Researcher, 29, 431-440.

FINCH, A. (2008). An Attitudinal Profile of EFL Learners in Korea. Electronic Journal of Foreign Language Teaching, 5(2), 206-219.

FLOWERDEW, J., MILLER, L. y LI, D. (2000). Chinese lecturers' perceptions, problems and strategies in lecturing in English to Chinese-speaking students. RELC Journal, 31, 116-138.

HOFSTEDE, G. et alii (2010). Cultures and Organizations: Software of the mind. Intercultural cooperation and its importance for survival (Revised and expanded third edition). Nueva York: McGraw-Hill.

HONEY, P. y MUMFORD, A. (1986). The manual of learning styles. Maidenhead: Peter Honey. 
HSIEH, S. Y (2015). La Vulnerabilidad de la propia imagen y la Búsqueda de la perfección lingüística. Condicionantes de la participación oral de los estudiantes taiwaneses en las clases de ELE. Revista Nebrija de Lingüística Aplicada a la Enseñanza de las Lenguas, 19, 1-16.

HSIEH, S. Y. (2016). La influencia de la percepción de la propia competencia lingüística en la participación en la clase de ELE. MarcoELE, 22, 1-21.

JACKSON, J. (2003). Case-based learning and reticence in a bilingual context: perceptions of business students in Hong Kong. System, 34(1), 457-469.

JENKINS, J. (2008). Taiwanese private university EFL students' reticence in speaking English. Taiwan Journal of TESOL, 5(1), 61-93.

JONES, J. F. (1999). From Silence to Talk: Cross-Cultural Ideas on Students' Participation in Academic Group Discussion. English for Specific Purposes, 18(3), 243-259.

JOY, S. y KOLB, D. A. (2008). Are there any cultural differences in learning style?. International Journal of Intercultural Relations, 33, 69-85.

KEATEN, J. et alii (1997). Development of an instrument to measure reticence. Communication Quarterly, 45(1), 37-54.

KEATEN, J. y KELLY, L. (2000). Reticence: An Affirmation and Revision». Communication Education, 49(2), 165-177.

KELLY, L. et alii (2010). Effects of reticence, affect for communication channels, and selfperceived competence on usage of instant messaging. Communication Research Reports, 27(2), 131-142.

KENNEDY, P. (2002). Learning cultures and learning styles: myth-understandings about adult (Hong Kong) Chinese learners. International Journal of Lifelong Education, 21(5), 430-445.

KOLB, D. A. (1984). Experiential learning: Experience as the source of learning and development. Nueva Jersey: Prentice-Hall.

LITTLEWOOD, W. (2000). Do Asian students really want to listen and obey?. ELT Journal, 54(1), 31-34.

LIU, M. (2005). Reticence in Oral English Language Classrooms: A Case Study in China. TESL Reporter, 38(1), 1-16.

LIU, N. y LITTLEWOOD, W. (1997). Why do many students appear reluctant to participate in classroom learning discourse?. System, 25(3), 331-384.

LOH, C. Y. R. y TEO, T. C. (2017). Understanding Asian Students Learning Styles, Cultural Influence and Learning Strategies. Journal of Education \& Social Policy, 7(1), 194210.

LUENGO-CERVERA, E. (2015). Learning Styles and Multiple Intelligences in the teaching-learning of Spanish as a foreign language. Enseñanza \& Teaching, 33(2), 79-103.

MANTIRI, O. (2013). The influence of culture on learning styles. SSRN Electronic Journal, 2566117, 1-16.

MARTÍNEZ, I. (2012). Explorar los estilos de aprendizaje del estudiante japonés: una posible clave para mejorar la didáctica del español en Japón. En F. GUERRA et. alii (eds.). Estilos de aprendizaje: investigaciones y experiencias (V Congreso Mundial de Estilos de Aprendizaje). Santander: Universidad de Cantabria. 
MITSIS, A. y FOLEY, P. (2004). The Effect of Students' Cultural Values on their StudentDriven Learning Preference. Working paper series 6/2004. Melbourne: Victoria University of Technology.

MORERA, I. (2016). Cultura y Estilos de Aprendizaje en la clase de español para inmigrantes [tesis doctoral]. Lovaina (Bélgica): Universidad de Lovaina.

MENDOZA, J. D. (2016). La reticencia a hablar en las clases de español en Corea. Un acercamiento a sus causas. MarcoELE, 22, 1-37.

MENDOZA, J. D. (2017). Estudiantes coreanos de español y renuencia a hablar en clase. ¿Realidad o mito?. Porta Linguarum, 28, 157-170.

MENDOZA, J. D. (2021). Razones de la renuencia a hablar en la clase de ELE en Asia Oriental. Análisis cualitativo y comparación entre estudiantes taiwaneses y coreanos. Tejuelo, 33, 39-74.

MUÑOZ TORRES, M. (2010a). Ansiedad en las actividades de aula: percepciones de los estudiantes chinos de español, SinoELE, 2, 1-14.

MUÑOZ TORRES, M. (2010a). Influencia del tipo de actividad en la ansiedad en alumnos chinos de español como lengua extranjera [trabajo final de máster]. España: Instituto Cervantes-UIMP.

MUSYTARIF, K. (2015). Learners' reasons for being reticent in speaking class [trabajo final de grado]. Mataram (Indonesia): Universidad de Mataram.

O' CONNELL, M. (2010). To text or not to text: Reticence and the utilization of short message services. Human Communication, 13(2), 87-102.

PHILLIPS, G. M. (1965). The problem of reticence. Pennsylvania Speech Annual, 22, 2238.

REID, J. M. (1987). The learning style preferences of ESL students. TESOL Quarterly, 21, 87-111.

SHIMIZU, J. (2006). Why are Japanese Students Reluctant to Express Their Opinions in the Classroom?. The Hiyoshi review of English studies, 48, 33-45.

SO0, R. S. y GOH, H. S. (2013). Reticent Students in the ESL Classroom. Advances in Language and Literary Studies, 4(2), 65-73.

SRIVORANART, P. (2015). Enseñanza adaptada al estilo de aprendizaje de los alumnos: estilo reflexivo y actividades para desarrollar el pensamiento crítico. En Y. MORIMOTO, M. V. PAVÓN y R. SANTAMARÍA (eds.). La enseñanza de ELE centrada en el alumno (Actas del XXV Congreso Internacional de ASELE). ASELE, 933-942.

SUGAHARA, S. y BOLAND, G. (2010). The Role of Cultural Factors in the Learning Style Preferences of Accounting Students: A Comparative Study between Japan and Australia». Accounting Education, 19(3), 235-255.

SWAIN, M. (1995). Three functions of output in second language learning». En G. COOK y B. SEIDLHOFER (eds.). Principle and Practice in Applied Linguistics. Oxford. Oxford University Press, 125-144.

SYED, M. (2006). Learning style among multi-ethnic students in four selected tertiary institutions in the Klang Valley [tesis doctoral]. Serdang (Malasia): Universidad Putra Malasia.

TALLEY, P. y TU, H. (2015). Reticence in the EFL Classroom: A Study in a Taiwanese EFL Context. Reino Unido: LAP Lambert. 
VOLET, S. y RENSHAW, P. (1996). Chinese students at an Australian university: adaptability and continuity. En D. A. WATKINS y J. B. BIGGS (eds.). The Chinese learner: cultural, psychological and contextual influences. Melbourne. ACER, 205220.

WU, H. (2019). Reticence in the EFL Classroom: Voices from Students in a Chinese University. International Journal of Applied Linguistics \& English Literature, 8(6), 114-125.

XIA, S. (2009). Are They Ready to Participate? East Asian Students' Acquisition of Verbal Participation in American Classrooms. Issues in Applied Linguistics, 17(2), 137-157.

XIAO, L. (2007). Bridging the Gap between Teaching Styles and Learning Styles: A Crosscultural Perspective. The Journal of Asia TEFL, 4(1), 55-78.

ZOLNAY, F. (2013). Estilos de aprendizaje, estilos de estudiantes y sus preferencias en cuanto a las actividades en la clase de ELE en la enseñanza bilingüe. En A. BLAS et alii (eds.). Actas del I Congreso Internacional de Didáctica de Español como Lengua Extranjera del Instituto Cervantes de Budapest. Budapest. Instituto Cervantes. 